\title{
Erratum to: Flavonoids mixture (diosmin, troxerutin, hesperidin) in the treatment of acute hemorrhoidal disease: a prospective, randomized, triple-blind, controlled trial
}

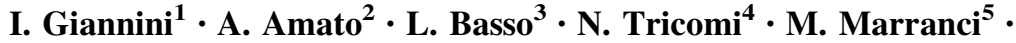

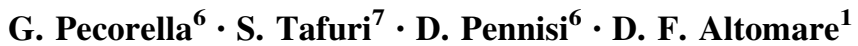

Published online: 4 September 2015

(C) Springer-Verlag Italia Srl 2015

\section{Erratum to: Tech Coloproctol (2015) 19:339-345 \\ DOI 10.1007/s10151-015-1302-9}

Unfortunately, the online published article has errors in Figure 1. The correct figure is given in the following page (Fig. 1).

The online version of the original article can be found under doi:10.1007/s10151-015-1302-9.

I. Giannini

ivanagi83@yahoo.it

D. F. Altomare

donatofrancesco.altomare@uniba.it

1 General Surgery and Liver Transplantation Unit, Department of Emergency and Organ Transplantation, "A. Moro"

University, Policlinico. Piazza G. Cesare, 11, 70124 Bari, Italy

2 Department of Coloproctology, Sanremo Hospital, Sanremo, Italy

3 Department of Surgery "P.Valdoni", Policlinico Umberto I ${ }^{\circ}$ "Sapienza" University, Rome, Italy

4 Coloproctology Unit, "Casa di Cura Candela", Palermo, Italy

5 Department of Surgery, "Casa di Cura Villa Fiorita", Prato, Italy

6 Department of Surgery, Policlinico Vittorio Emanuele, University of Catania, Catania, Italy

7 Department of Biomedical Science and Human Oncology, "A. Moro" University, Bari, Italy 
Fig. 1 CONsolidated Standard of Reporting Trials flow diagram

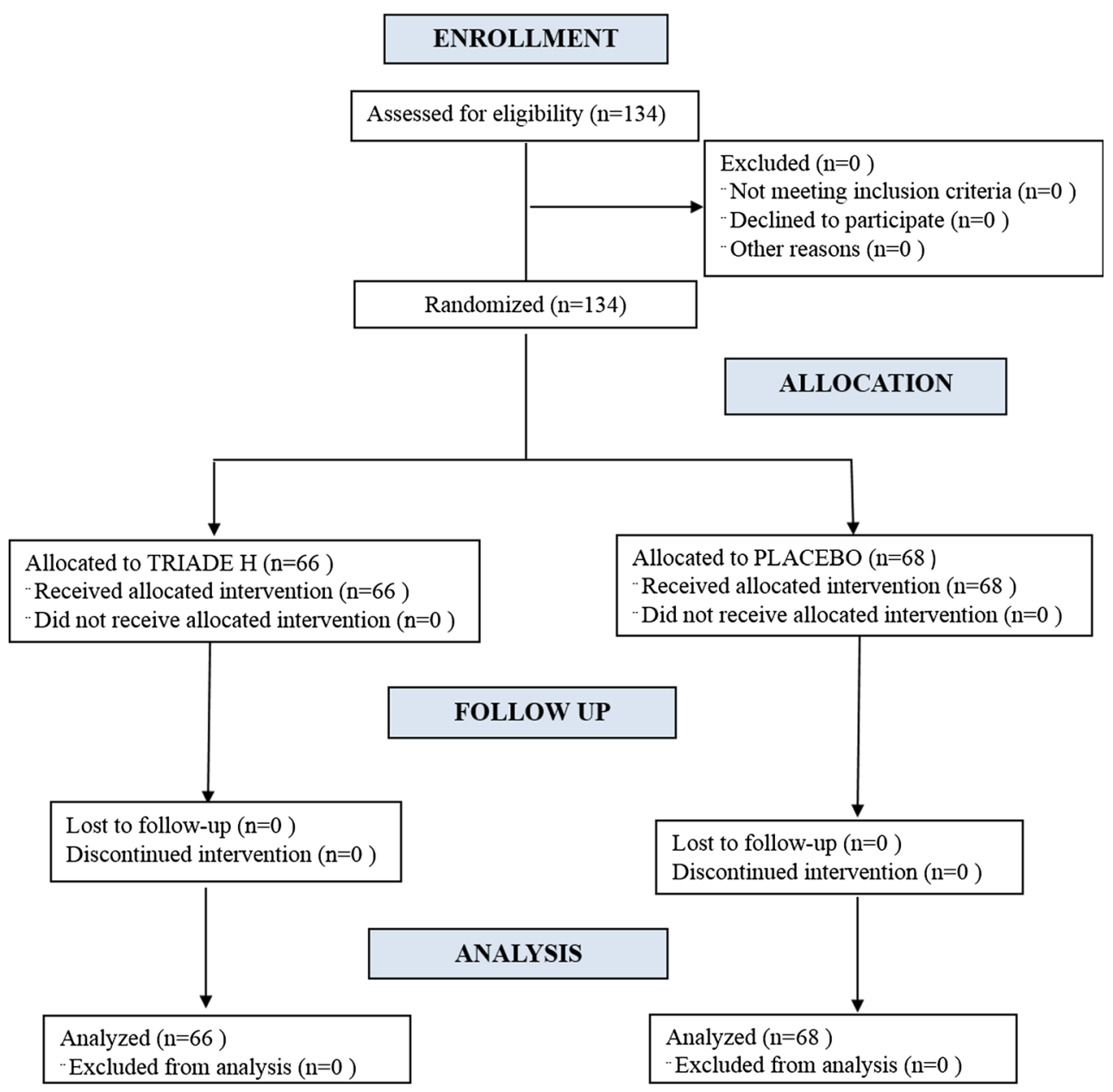

